- Original Article

\title{
Reliability and Validity of an Ultrasonic Device for Measuring Height in Adults
}

\author{
Seon Hwa Cho, Young Gyu Cho*, Hyun Ah Park, A Ra Bong \\ Department of Family Medicine, Inje University Seoul Paik Hospital, Inje University College of Medicine, Seoul, Korea
}

Background: The ultrasonic stadiometer was originally developed as a device to measure and monitor children's height. However, an ultrasonic stadiometer (InLab S50; InBody Co., Seoul, Korea) was used to measure adults' height in the 2018 Korea Community Health Survey (KCHS). This study was conducted to assess the reliability and validity of the InLab S50 in adults.

Methods: The study subjects were 120 adults (reliability test, $\mathrm{n}=20$; validity test, $\mathrm{n}=100$ ) who had visited a health screening center. The intra- and inter-rater reliabilities of InLab S50 were assessed using the intraclass correlation coefficient (ICC). The agreement between InLab S50 and an automatic stadiometer (HM-201; Fanics, Busan, Korea) was assessed using Pearson's correlation coefficient and Bland-Altman analysis.

Results: The intra- and inter-rater reliabilities of the InLab S50 were excellent (ICC $=0.9999$ and 0.9998 , respectively). The correlation coefficient of the height measured by the two measurement devices was very high ( $\mathrm{r}=0.996)$. The difference ( $\Delta$ height [HM-201-InLab S50]) was $-0.15 \pm 0.78 \mathrm{~cm}$ (95\% limit of agreement [LOA], -1.69 to 1.38). After excluding the values outside $95 \% \mathrm{LOA}$, the difference was further reduced to $-0.05 \pm 0.59 \mathrm{~cm}$ ( $95 \% \mathrm{LOA},-1.20$ to $1.10)$.

Conclusion: This study showed that the InLab S50 is a reliable and valid device for the measurement of adults' height. Therefore, we think that InLab S50 could be used to measure adults' height in household health surveys such as the KCHS.

Keywords: Body Height; Ultrasonic Waves; Health Surveys; Validation Study

Received: September 1, 2020, Revised: September 28, 2020, Accepted: October 8, 2020

${ }^{*}$ Corresponding Author: Young Gyu Cho https://orcid.org/0000-0003-1017-8884

Tel: +82-2-2270-0097, Fax: +82-2-2267-2030, E-mail: jacobel@paik.ac.kr 


\section{INTRODUCTION}

Obesity is defined as abnormal body fat accumulation, associated with an increased risk of type 2 diabetes, cancer, cardiovascular diseases, and mortality. ${ }^{1)}$ Obesity and comorbidities related to obesity can lead to an increase in socioeconomic burden. ${ }^{2)}$ Despite the increase in public awareness about the harmful impact of obesity, and the development and implementation of national comprehensive policies to tackle obesity, it has been increasing among Korean adults. ${ }^{3)}$ Most health organizations have adopted body mass index (BMI) as a clinical indicator to diagnose obesity. The Korean Society for the Study of Obesity defines a BMI of $25-29.9 \mathrm{~kg} / \mathrm{m}^{2}$ as pre-obese and a BMI $\geq 30 \mathrm{~kg} / \mathrm{m}^{2}$ as obese. $^{4)}$

BMI is calculated by dividing body weight $(\mathrm{kg})$ by height squared $\left(\mathrm{m}^{2}\right)$. Numerous epidemiological studies have calculated BMI using self-reported height and weight. These studies have shown that individuals tend to over-report their height and under-report their weight, resulting in an underestimated BMI. ${ }^{5)}$ The Korean national prevalence of obesity is officially estimated using data from the Korea National Health and Nutrition Examination Survey (KNHANES) ${ }^{6}{ }^{6}$ In 2008, the Korea Community Health Survey (KCHS), another nationwide survey, was initiated to generate community health statistics. ${ }^{7)}$ While anthropometric measurements in KNHANES were performed using certified equipment by trained medical personnel, the data of height and weight in KCHS were collected through a self-report method by trained interviewers from 2008 to 2017. Park et al. ${ }^{8}$ reported that KCHS 2010, using self-reported BMI, underestimated the prevalence of obesity by $8.6 \%$ compared to KNHANES 2010 using measured BMI. Accurate monitoring of obesity is necessary to implement effective community health policies. Since it was widely acknowledged that there was a large bias in self-reported BMI, anthropometric measurements using mechanical devices were introduced in the KCHS in 2018.

While the health examination in KNHANES was performed at a mobile examination center, KCHS was conducted by visiting individuals at their homes. Because examiners are required to visit the selected sample households with their measuring devices, the devices had to be small, lightweight, and portable. The ultrasonic stadiometer is a device used to measure the distance from the top of a person's head to the floor using an ultrasonic wave. Although the ultrasonic stadiometer has been used to measure and monitor children's height, ${ }^{9,10)}$ an ultrasonic device (InLab S50; InBody Co., Seoul, Korea) can measure up to $200.0 \mathrm{~cm} .{ }^{11)}$ Owing to its wide range, the KCHS committee supposed that the ultrasonic stadiometer could also be used to measure adults' height. A preliminary study with 14 adults was conducted to evaluate the accuracy of the InLab S50. As a result, InLab S50 overestimated height by $0.44 \mathrm{~cm}$ compared to an automatic stadiometer (DS-102; Dongsahn Jenix Co., Seoul, Korea). Although the committee officially adopted InLab S50 as the device to measure heights in KCHS, they suggested that the reliability and validity of InLab S50 should be reevaluated with a large sample.

This study was conducted to assess the reliability and validity of an ultrasonic stadiometer in adults compared to an automatic stadiometer. If a measurer is shorter than a subject, it is difficult to handle an ultrasonic device on the head. Therefore, we assessed whether using a footstool would affect the reliability and validity of the device.

\section{METHODS}

\section{Study Subjects}

Korean adults aged 19 years or older who visited the Seoul Paik Hospital Health Promotion Center for health checkups were recruited during January-February, 2020. Twenty adults volunteered for a test to assess the intra- and inter-rater reliability of InLab S50, while 100 adults applied for a test to assess the agreement between InLab S50 and an automatic stadiometer (HM-201; Fanics, Busan, Korea). After completing the study, a gift certificate with a value of 10,000 Korean won was offered to each participant. All study subjects provided written informed consent to participate in the study. The study protocol was reviewed and approved by the Institutional Review Board of Seoul Paik Hospital (IRB approval no., PAIK 2019-11-008).

\section{Measurements}

InLab S50 is a handheld height-measuring device that uses ultrasound technology. The device is triangular-shaped with an ultrasound sensor at the bottom, an liquid-crystal display (LCD) screen, and a button on top. The height of the subjects was measured using InLab S50 following the manufacturer's instructions. ${ }^{11)}$ The subjects were required to stand up straight without shoes against the wall on a solid and flat floor. InLab S50 was then placed on the subject's head and maintained at a horizontal level. A customized adjunct was applied to help the InLab S50 maintain a horizontal level. It was ensured that there were no obstacles between the ultrasound sensor and the foor. The height was displayed to the nearest $0.1 \mathrm{~cm}$ on the LCD screen.

In the reliability test, two measurers (A and B) performed height measurements using InLab S50. Measurer A (S.H.C.) first measured the height of the subjects twice, then measurer B (A.R.B.) measured their height once in the same manner. Subsequently, measurers A and $B$ repeated the same height measurement process while they stood on a footstool. The footstool used in this study was plastic, about $19.0 \mathrm{~cm}$ in width, $22.5 \mathrm{~cm}$ in length, and $19.0 \mathrm{~cm}$ in height and can be easily purchased on any market such as Daiso.

In the validity test, measurer A performed height measurement using two types of stadiometers (InLab S50 and HM-201). HM-201, an automatic stadiometer, which is standardized and widely used in health facilities, was used as the reference device. HM-201 was calibrated by the manufacturer before this study. Measurer A measured the height of the subjects using InLab S50 on the floor, followed by standing on a footstool. After that, their heights were measured again using HM-201.

\section{Statistical Analyses}

Data are expressed as mean \pm standard deviation or number (\%). The 
intra- and inter-rater reliabilities were the degree of agreement between the heights measured twice by measurer $A$ and between the height first measured by measurer A and measured by measurer B, respectively. The intra- and inter-rater reliabilities of the InLab S50 were assessed using the intraclass correlation coefficient (ICC). The agreement between InLab S50 and HM-201 was assessed using Pearson's correlation coefficient and Bland-Altman analysis. All statistical analyses were performed using PASW SPSS ver. 18.0 for Windows (SPSS Inc., Chicago, IL, USA). Statistical significance was set at $\mathrm{P}<0.05$.

\section{RESULTS}

\section{Reliability Test}

A total of 20 adults (six men and 14 women) participated in the reliability test of InLab S50, and their mean age was 31.8 \pm 15.7 years (Table 1). The mean heights of the subjects first measured and remeasured by measurer A, were $163.99 \pm 6.70 \mathrm{~cm}$ and $163.98 \pm 6.72 \mathrm{~cm}$, respectively. The mean height of the subjects measured by measurer B was $163.98 \pm$ $6.73 \mathrm{~cm}$.

The intra-rater reliability of the InLab S50 was excellent (ICC= 0.9999). The absolute mean difference between the heights of the subjects first measured and remeasured was $0.01 \pm 0.11 \mathrm{~cm}$ and the maximal difference was $0.2 \mathrm{~cm}$ (Table 2). The inter-rater reliability of the InLab S50 was also excellent (ICC=0.9998). The absolute mean difference between the heights of the subjects measured by measurer $A$ and

Table 1. General characteristics of the study subjects

\begin{tabular}{lcc}
\hline Characteristic & Reliability test $(\mathrm{n}=20)$ & Validity test $(\mathrm{n}=100)$ \\
\hline Age $(\mathrm{y})$ & $31.8 \pm 15.7$ & $51.0 \pm 17.2$ \\
Age group $(\mathrm{y})$ & & \\
$19-39$ & $15(75.0)$ & $23(23.0)$ \\
$40-59$ & $3(15.0)$ & $44(44.0)$ \\
$\geq 60$ & $2(10.0)$ & $33(33.0)$ \\
Sex & & \\
Men & $6(30.0)$ & $49(49.0)$ \\
Women & $14(70.0)$ & $51(51.0)$ \\
\hline
\end{tabular}

Values are presented as mean \pm standard deviation or number (\%).
B was $0.005 \pm 0.128 \mathrm{~cm}$ and the maximal difference was $0.2 \mathrm{~cm}$ (Table 3).

Reliability of InLab S50 was reassessed when measurers A and B performed height measurements using a footstool. All ICCs of intrarater reliability (0.9999) and inter-rater reliability (0.9996) of InLab S50 were excellent even when using a footstool (Tables 2, 3).

\section{Validity Test}

A total of 100 adults ( 49 men and 51 women) participated in the validity test of InLab S50, and their mean age was 51.0 \pm 17.2 years (Table 1). The mean heights of the subjects measured using InLab S50 while standing on the floor and on a footstool were $164.41 \pm 8.76 \mathrm{~cm}$ and $164.36 \pm 8.76 \mathrm{~cm}$, respectively. The mean height of the subjects measured using HM-201 was $164.26 \pm 8.80 \mathrm{~cm}$.

The correlation between the heights measured using HM-201 and InLab S50 was highly significant ( $\mathrm{r}=0.996)$ (Figure 1A). The difference between the two measurements ( $\triangle$ height [HM-201-InLab S50]) was $-0.15 \pm 0.78 \mathrm{~cm}$ ( $95 \%$ limits of agreement [LOA], -1.69 to 1.38 ). This difference was consistent across the range of measurements. Outliers above the upper LOA were not found, but outliers below the lower LOA were found in four subjects (Figure 1B). Excluding these four subjects' data, the difference was reduced to $-0.05 \pm 0.59 \mathrm{~cm}(95 \% \mathrm{LOA}$, -1.20 to 1.10 ).

The validity of the InLab S50 was reassessed when height measurements were performed after standing on a footstool. The correlation between the heights measured using HM-201 and InLab S50 with a footstool was also highly significant $(\mathrm{r}=0.996)$ (Figure $2 \mathrm{~A})$. The difference between the two measurements was $-0.10 \pm 0.77 \mathrm{~cm}(95 \% \mathrm{LOA}$, -1.62 to 1.42) when using a footstool, which was consistent throughout the range of measurement. Although outliers above the upper LOA were found in one subject, outliers below the lower LOA were found in four subjects (Figure 2B). Excluding these five subjects' data, the difference was further reduced to $-0.03 \pm 0.60 \mathrm{~cm}$ (95\% LOA, -1.21 to 1.15$)$.

\section{DISCUSSION}

This study aimed to assess the reliability and validity of InLab S50 in

Table 2. Intra-rater reliability of height measured using InLab S50 ( $n=20)$

\begin{tabular}{ccccc}
\hline Variable & Test & Retest & ICC $(95 \%$ Cl) & P-value \\
\hline On the floor & $163.99 \pm 6.70$ & $163.98 \pm 6.70$ & $0.9999(0.9996-0.9999)$ & $<0.001$ \\
On a footstool & $164.03 \pm 6.69$ & $163.99 \pm 6.72$ & $0.9999(0.9996-0.9999)$ & $<0.001$ \\
\hline
\end{tabular}

Values are presented as mean \pm standard deviation, unless otherwise stated.

ICC, intraclass correlation coefficient; $\mathrm{Cl}$, confidence interval.

Table 3. Inter-rater reliability of height measured using InLab S50 ( $\mathrm{n}=20)$

\begin{tabular}{ccccc}
\hline \multicolumn{1}{c}{ Variable } & Measurer 1 & Measurer 2 & ICC (95\% Cl) & P-value \\
\hline On the floor & $163.99 \pm 6.70$ & $163.98 \pm 6.73$ & $0.9998(0.9995-0.9999)$ & $<0.001$ \\
On a footstool & $164.03 \pm 6.69$ & $164.02 \pm 6.73$ & $0.9996(0.9989-0.9998)$ & $<0.001$ \\
\hline
\end{tabular}

Values are presented as mean \pm standard deviation, unless otherwise stated.

ICC, intraclass correlation coefficient; $\mathrm{Cl}$, confidence interval. 

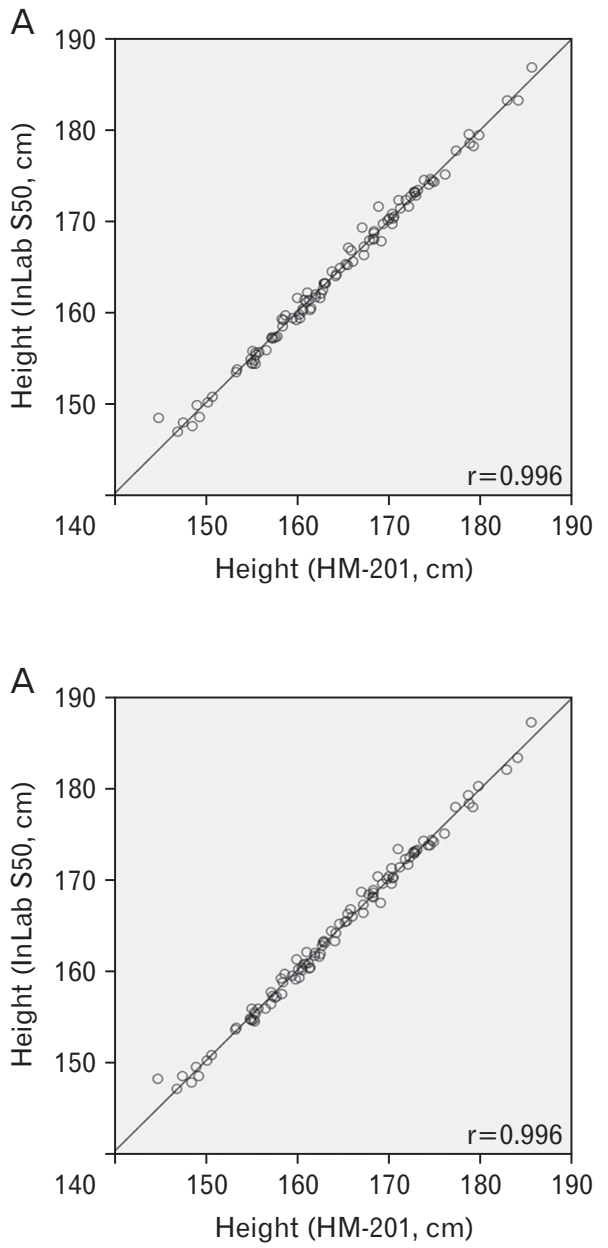

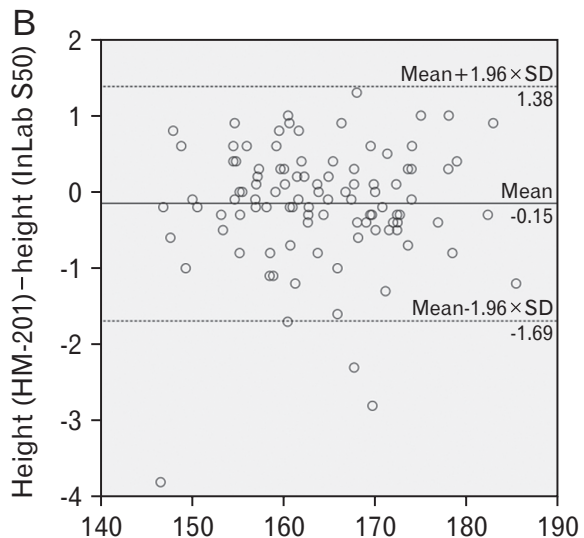

[Height (HM-201)+height (InLab S50)]/2

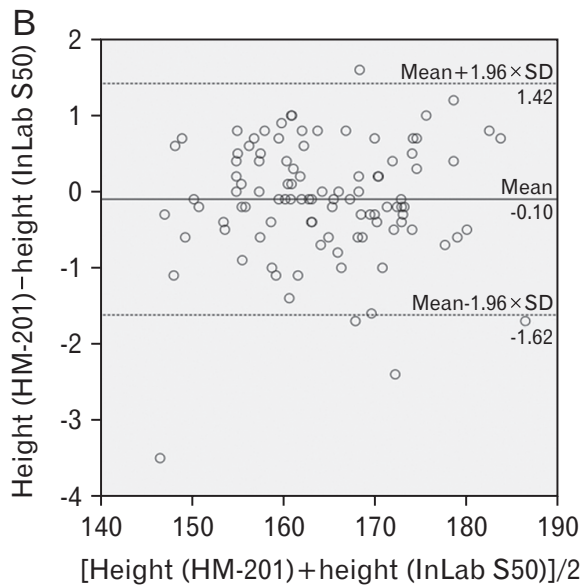

Figure 1. Scatter plot (A) and Bland-Altman plot (B) of height measured using HM-201 and InLab S50 on the floor. SD, standard deviation.
Figure 2. Scatter plot (A) and Bland-Altman plot (B) of height measured using HM-201 and InLab S50 standing on a footstool. SD, standard deviation. adults. We found that the intra- and inter-rater reliability of InLab S50 (ICC $=0.9999$ and 0.9998 , respectively) were excellent and there was a very good agreement between InLab S50 and HM-201 ( $\Delta$ height [HM201 -InLab S50] $=-0.15 \pm 0.78 \mathrm{~cm}$ ). These findings indicate that InLab S50 can be used as a tool to measure adults' height in epidemiological surveys such as the KCHS.

The ultrasound stadiometer was originally developed as a device for parents to measure and monitor their children's height. Watt et al. ${ }^{9)}$ reported that an ultrasound stadiometer (Gulliver; Syrinx-Diagnosika $\mathrm{GmbH}$, Frankfurt, Germany) underestimated height by approximately $0.5 \mathrm{~cm}$ and was less repeatable in measuring children's heights compared to a conventional stadiometer (Harpenden stadiometer). In their study, they concluded that Gulliver was unlikely to replace a standard mechanical stadiometer, although it was novel, portable, and simple to use. In contrast, Glock et al. ${ }^{10)}$ reported that there was no significant difference between the children's height measured using the Gulliver and Harpenden stadiometers. Therefore, they suggested that Gulliver could produce accurate results in children's height measurements. As our study was performed in adults and a different ultrasonic device (InLab S50) was used, our findings could not be directly compared to those of previous studies. To our knowledge, this is the first study to assess the reliability and validity of an ultrasonic stadiometer in adults.

In this study, the difference between HM-201 and InLab S50 ( $\Delta$ height [HM-201-InLab S50]) was merely $-0.15 \mathrm{~cm}$. After excluding values outside $95 \% \mathrm{LOA}$, the difference was further reduced to $-0.05 \mathrm{~cm}$. While there were no outliers above the upper LOA, outliers below the lower LOA were found in four subjects. This result indicates that InLab S50 overestimated heights in a few subjects compared to HM-201, which may have caused significant measurement bias. The largest difference $(-3.8 \mathrm{~cm})$ was observed in a 75 -year-old woman. She had a forward bending posture and trouble straightening her back. While she could stand up against the wall when measuring her height using InLab S50, it was more difficult for her back to be completely against a narrow backboard while using HM-201. The measurement bias could have been caused not only by inaccurate devices but also by the improper positions of subjects. ${ }^{12)} \mathrm{HM}-201$ was used as a reference device to assess the validity of InLab S50, but it does not imply that HM-201 can measure the height without error. ${ }^{13,14)}$ It is more likely that HM-201 underestimated her height compared to InLab S50 due to improper positioning during the height measurement using HM-201. The other three outliers (49-year-old man, 62-year-old man, and 54-year-old 
woman) had no apparent abnormality in appearance. We cannot clearly explain why there was a larger bias in their height measurements, but we think it is because measurement protocol deviation might have occurred unintentionally when measuring their heights.

To assess the accuracy of InLab S50, it would be more appropriate to exclude subjects from the study who could not comply with the measurement protocol. However, this study was originally designed to assess whether InLab S50 is accurate enough to be used to measure adults' height in the KCHS. The KCHS sample includes even the elderly who cannot stand upright for height measurement. ${ }^{7)}$ Therefore, we did not make any exclusion criteria other than age in this study. It is generally assumed that height does not change during adulthood. However, height begins to decrease by age 30 and height loss accelerates with advancing age. ${ }^{15)}$ Height declines, on an average, by 2 to $4 \mathrm{~cm}$ over the course of an adult's life. ${ }^{16)}$ Age-related height loss is largely attributable to vertebral deformity through degenerative changes of the vertebral body and intervertebral disc. ${ }^{17)}$ Moreover, as the elderly have fewer opportunities to measure their heights, they are more prone to overreporting them. ${ }^{18)}$ Although there are some elderly who cannot adhere to the height measurement protocol, the introduction of InLab S50 in KCHS is expected to contribute to the resolution of inaccuracy of self-reported height in the elderly.

Additionally, we reassessed the reliability and validity of the InLab S50 when height measurements were performed using a footstool. The reliability of InLab S50 was still excellent and the difference between HM-201 and InLab S50 ( $\Delta$ height [HM-201-InLab S50]=$0.10 \pm 0.77 \mathrm{~cm}$ ) decreased slightly when using a footstool. The LCD screen was located on the top of InLab S50. Therefore, when a measurer with short stature tries to measure the height of a taller subject using InLab S50, measurement bias may increase and physical contact may occur. However, using a footstool can help perform a more accurate and safe height measurement in adults.

This study had several limitations. The height measurement was performed by only two measurers in only one health facility. Usually, a hospital provides a stable environment for measuring the height. However, in KCHS, the height measurement was performed by a number of measurers in various environments. The bias in height measurement may vary depending on the environment and competence of measurers. The KCHS committee should develop a height measurement manual applicable to various environments and provide all measurers with a proper training program for the manual. We did not exclude subjects who could not comply with the measurement protocol. However, this was because the KCHS does not exclude them either. If subjects with height deformities had been excluded, the bias of InLab S50 would have reduced further. In the validity test, the order of the measurement is important. If a reference device is initially used for measuring the subjects' height, the measurer might have a tendency to approximate the initial results when remeasuring with an experimental device. Therefore, we first measured the heights with InLab S50 and then HM-201 was used as a reference device.

Accurate height measurement is an integral part of community health monitoring. This study showed that InLab S50 is a reliable and valid device for measuring adults' height. Therefore, we think that InLab S50 can be used to measure adults' height in household health surveys such as the KCHS.

\section{CONFLICT OF INTEREST}

No potential conflict of interest relevant to this article was reported.

\section{FUNDING}

This work was supported by funds from the Korea Centers for Disease Control and Prevention.

\section{ORCID}

Seon Hwa Cho: https://orcid.org/0000-0002-5706-7738

Young Gyu Cho: https://orcid.org/0000-0003-1017-8884

Hyun Ah Park: https://orcid.org/0000-0003-2343-8964

A Ra Bong: https://orcid.org/0000-0001-8749-1727

\section{REFERENCES}

1. Guh DP, Zhang W, Bansback N, Amarsi Z, Birmingham CL, Anis AH. The incidence of co-morbidities related to obesity and overweight: a systematic review and meta-analysis. BMC Public Health 2009;9:88.

2. Kjellberg J, Tange Larsen A, Ibsen R, Hojgaard B. The socioeconomic burden of obesity. Obes Facts 2017;10:493-502.

3. Yoon JH. Prevalence of obesity and incidence of obesity-related comorbidities in Koreans based on National Health Insurance Service Health Checkup Data 2006-2015 (J Obes Metab Syndr 2018;27:46-52). J Obes Metab Syndr 2018;27:195-7.

4. Seo MH, Lee WY, Kim SS, Kang JH, Kang JH, Kim KK, et al. 2018 Korean Society for the Study of Obesity guideline for the management of obesity in Korea. J Obes Metab Syndr 2019;28:40-5.

5. Ng CD. Biases in self-reported height and weight measurements and their effects on modeling health outcomes. SSM Popul Health 2019;7: 100405.

6. Kweon S, Kim Y, Jang MJ, Kim Y, Kim K, Choi S, et al. Data resource profile: the Korea National Health and Nutrition Examination Survey (KNHANES). Int J Epidemiol 2014;43:69-77.

7. Kang YW, Ko YS, Kim YJ, Sung KM, Kim HJ, Choi HY, et al. Korea Community Health Survey data profiles. Osong Public Health Res Perspect 2015;6:211-7.

8. Park YR, Cho YG, Kang JH, Park HA, Kim KW, Hur YI, et al. Comparison of obesity and overweight prevalence among Korean adults according to Community Health Survey and Korea National Health and Nutrition Examination Survey. Korean J Obes 2014;23:64-8.

9. Watt V, Pickering M, Wales JK. A comparison of ultrasonic and mechanical stadiometry. Arch Dis Child 1998;78:269-70.

10. Glock M, Hermanussen M, Keller E, Hartmann KK. Gulliver G-100: a new device to evaluate daily growth measurement in comparison with Harpenden stadiometer. Horm Res 1999;52:287-90. 
11. InBody. InBody the pioneer [Internet]. Seoul: InBody Co. Ltd.; [date unknown] [cited 2020 Feb 25]. Available from: https://www.inbody. com/global/product/InLab.aspx.

12. Madden AM, Smith S. Body composition and morphological assessment of nutritional status in adults: a review of anthropometric variables. J Hum Nutr Diet 2016;29:7-25.

13. Bland JM, Altman DG. Measuring agreement in method comparison studies. Stat Methods Med Res 1999;8:135-60.

14. Kong KA. Statistical methods: reliability assessment and method comparison. Ewha Med J 2017;40:9-16.

15. Sorkin JD, Muller DC, Andres R. Longitudinal change in height of men and women: implications for interpretation of the body mass index: the Baltimore Longitudinal Study of Aging. Am J Epidemiol 1999;150: 969-77.

16. Fernihough A, McGovern ME. Physical stature decline and the health status of the elderly population in England. Econ Hum Biol 2015;16: 30-44.

17. Huang W, Lei X, Ridder G, Strauss J, Zhao Y. Health, height, height shrinkage, and SES at older ages: evidence from China. Am Econ J Appl Econ 2013;5:86-121.

18. Frid H, Adolfsson ET, Rosenblad A, Nydahl M. Agreement between different methods of measuring height in elderly patients. J Hum Nutr Diet 2013;26:504-11. 